LPTENS-03/22

DFTT $14 / 03$

\title{
Twisted determinants on higher genus Riemann surfaces
}

\author{
Rodolfo Russo $^{a}$ and Stefano Sciuto ${ }^{b}$ \\ a Laboratoire de Physique Théorique de l'Ecole Normale Supérieure, \\ 24 rue Lhomond, F-75231 Paris Cedex 05, France \\ b Dipartimento di Fisica Teorica, Università di Torino; \\ I.N.F.N., Sezione di Torino, Via P. Giuria 1, I-10125 Torino, Italy \\ rodolfo.russo@lpt.ens.fr, sciuto@to.infn.it
}

\begin{abstract}
We study the Dirac and the Laplacian operators on orientable Riemann surfaces of arbitrary genus $g$. In particular we compute their determinants with twisted boundary conditions along the $b$-cycles. All the ingredients of the final results (including the normalizations) are explicitly written in terms of the Schottky parametrization of the Riemann surface. By using the bosonization equivalence, we derive a multi-loop generalization of the well-known $g=1$ product formulae for the Theta-functions. We finally comment on the applications of these results to the perturbative theory of open charged strings.
\end{abstract}




\section{Introduction}

The perturbative expansion of string theory has been thoroughly studied in the last thirty years and the first works date back to the time of the dual models [1]. Our understanding of this subject has greatly increased during the years, in particular in the eighties, with many conceptual and technical breakthroughs (see for instance [2]). The geometrical meaning of string amplitudes became much more clear and the strict relation of these physical quantities with the theory of Riemann surfaces and Theta-functions made the subject even more interesting.

Among the basic building blocks of string amplitudes one finds the determinants of the Laplacian and of the Dirac operator. Since these operators are present in the 2D action describing the free string propagation, their determinants will appear in all perturbative amplitudes, starting from the simplest one: the vacuum energy. A detailed study of these determinants was performed in the papers $[3,4,5,6,7,8]$.

It is particularly interesting to compare the fermionic and the bosonic results, because, even though they have a very different structure, they are related through the bosonization duality. Thus the equivalence between the fermionic and the bosonic determinants can be used as a tool for giving "physicist proofs" of interesting mathematical identities $[9,10,11]$. In fact it is known that from general addition theorems for Thetafunctions (see, for example, Proposition 2.16 of [9]) one can derive some identities proving the equivalence between bosonic and fermionic systems on a $g$-loop Riemann surface. For instance, the identity in Corollary 2.17 of [9] is a consequence (or a proof, according to the points of view) of the relation between a fermionic system ${ }^{1}(b, c)$ of $\operatorname{spin}(1,0)$ and a chiral boson of background charge $Q=-1$ :

$$
\theta\left(\sum_{i=1}^{g} J\left(z_{i}\right)-J(w)-\Delta \mid \tau\right) \frac{\prod_{i<j=1}^{g} E\left(z_{i}, z_{j}\right)}{\prod_{i=1}^{g} E\left(z_{i}, w\right)} \frac{\prod_{i=1}^{g} \sigma\left(z_{i}\right)}{\sigma(w)}=C \operatorname{det}\left[\omega_{i}\left(z_{j}\right)\right]
$$

In the mathematical literature, the analysis of identities like the one above focuses on the dependence of the various functions on the punctures $z_{i}$. Thus often these equalities are written in terms of some "constants" (like $C$ in the above equation) that depend only on the moduli of the surface, but not the punctures $z_{i}$. However, it is also quite interesting to give an explicit expressions of these constants, since they are closely related to the partition functions of the bosonic and fermionic systems. A powerful technique for writing explicitly $C$ in terms of the moduli of the surface is the sewing technique. This is a very old idea [1] allowing to construct higher loop amplitudes from tree diagrams: pairs of external legs are sewn together taking the trace over all possible states with the insertion of a propagator that geometrically identifies the neighborhoods around two punctures. The results obtained in this way give rise to a particular parametrization of the $g$-loop Riemann surface known as Schottky uniformization. At 1-loop, string theorists are very familiar with this parametrization: in this case, the torus is just seen as the complex plane where two points $z, w$ are identified if $z=k^{n} w, \forall n \in \mathbb{Z}$. Here $k$ is a

\footnotetext{
${ }^{1}$ See Appendix A for the definition of our conventions.
} 
complex parameter representing the modulus of the torus and is related to period matrix entering in the Theta-functions by $k=\exp (2 \pi \mathrm{i} \tau)$, with $\operatorname{Im} \tau>0 \Leftrightarrow|k|<1$. At 1 -loop the constant $C$ in Eq. (1.1) is just $C=\prod_{n=1}^{\infty}\left(1-k^{n}\right)^{3}$ and is related to the partition function of a chiral scalar field. This pattern can be generalized to higher genus surfaces by sewing other handles to the 1 -loop result and one gets $C=\prod_{\alpha}^{\prime} \prod_{n=1}^{\infty}\left(1-k_{\alpha}^{n}\right)^{3}$, where the exact meaning of $k_{\alpha}$ and of product over the Schottky group $\prod_{\alpha}^{\prime}$ will be explained later (see Eq. (2.11) and Appendix B). Here we just want to stress that identities like Eq. (1.1) can be exploited also to rewrite products over the Schottky group in terms of more geometrical quantities like Theta-function, Abelian differentials, and Prime form (see Appendix A).

At 1-loop, the possibility to pass from the formulation in terms of Schottky products to the one with Theta-functions has been heavily exploited in string theory. The physical reason is clear: the geometrical expressions in terms of Theta-functions has the advantage to make manifest the modular properties of the string results. This is in contrast to the expressions written in terms of Schottky products, where these properties are not manifest. In fact a modular transformation can be non-analytic in $k$ (for instance, consider $\tau \rightarrow-1 / \tau)$. This limitation is precisely the reason why the Schottky uniformization has not been much studied by mathematicians. However, the Schottky uniformization has an important physical significance because it makes manifest the unitarity properties of string amplitudes. This can be easily understood by remembering that this uniformization naturally arises from the sewing procedure where the Schottky multipliers $k$ are basically the equivalent of the (exponential of the) Schwinger parameters in field theory. Thus the Taylor expansion in the $k$ 's of the string results isolates the contribution to the amplitude coming from the propagation of particular states in the various handles of the surface. At 1-loop level, the relation between $k$ and $\tau$ is particularly simple and thus one can use also the Theta-function expressions to analyze the unitarity properties of the string results. However, on a $g$-loop surface there is no equivalent of the simple relation $k=\exp (2 \pi \mathrm{i} \tau)$ and only the expressions in terms of Schottky parameters display the unitarity properties in a simple way.

Thus the expressions of string results à la Schottky and the one in terms of Thetafunctions capture two different but equally important features of string theory. Depending on the question one would like to answer it is more convenient to write the amplitudes in one or the other form. Because of this, it is quite crucial to be able to rewrite a general Schottky product in terms of geometrical objects and vice-versa. As we already said, this step is by now standard in 1-loop computations, where the period matrix is just a complex number which is related in a very simple way to the only Schottky multiplier $k$. In this case, string theorists often make use of identities like the one in Eq. (3.3). At mathematical level, these 1-loop identity can be proved by showing that both sides of the equation have the same periodicity, poles and zeros. The generalization of this kind of identities to the higher loop case is much harder. However, as we noticed before, it is precisely in the multiloop expressions where one really needs to have these identities to pass from a manifest unitarity amplitude to a modular covariant one.

Thus the main purpose of this paper is to find new relations of the kind of Eq. (1.1) 
that relate Theta-functions to the Schottky products contained in the constant $C$. Following the ideas of $[14,15,16]$, we use the equivalence between bosonic and fermionic systems as a device allowing to recast Schottky products in terms of Theta-functions. We basically generalize previous analysis $[14,15,16]$ in two directions. On one hand we consider fermions of spin $(\lambda, 1-\lambda)$ and the dual bosonic system with the background charge $Q=1-2 \lambda$, instead of focusing just on the simplest case $\lambda=1 / 2$. On the other hand, for general $\lambda$ we also consider twisted periodicity conditions along the $b$-cycles. This twisting can be equivalently thought as the effect of a flat gauge connection along the $b$ - cycles $^{2}$ on a minimally coupled fermionic system. Thus the periodicity parameters $\left(\epsilon_{\mu}\right)$ can be naturally thought as non-geometrical parameters of the Riemann surface. In fact, in the generalization of Eq. (1.1) arising in these cases, the constants $C$ depend also on $\epsilon_{\mu}$ beyond the usual dependence on the geometrical moduli. Our goal is to give an explicit expressions in terms of Schottky series or products for all the quantities appearing in the various identities. In this way, at least in principle, one can compute all the ingredients in terms of the parameters of the surfaces with an arbitrary degree of precision.

One more clarification is due at this point, since it may appear unclear why in our study $b$-cycles twists have a privileged status in comparison with $a$-cycle twists. The difference is simply due to the Hamiltonian approach adopted here, where the twists along the time direction can be taken into account simply by a modification of the sewing propagator, while those along the spatial directions modifies radically the spectrum of the free theory. In the higher genus diagrams, this difference implies that $b$-cycles twists can be described à la Schottky by means of the usual representations of the projective group, while the direct description of amplitudes with $a$-cycle twists seems to require a more complicated formalism (for an explicit 1-loop example see [17] and compare Eqs. (7) and (8) therein). Of course, once a result with $b$-cycles twists is known in terms of Thetafunction, one can explicitly perform a modular transformation and derive the equivalent quantity with $a$-cycles twists. This strategy can be used to obtain the partition function of the charged open bosonic string in presence of a constant external field.

The structure of the paper is the following. In Section 2 we recall the main features of the sewing technique, focusing on the fermionic correlators. The presence of general twisted periodicity along the $b$-cycles requires a modification of the sewing procedure and it is crucial for the consistency of the results to carefully follow all the effects due to the presence of $\epsilon_{\mu}$. The main result of this section is the explicit expression (2.18) of the twisted abelian differentials. In Section 3 we compare the fermionic correlators with the corresponding bosonic ones and derive Eqs. (3.5)-(3.6) which generalize (1.1) to the twisted case. Finally in the Conclusions we discuss some applications of our results in the context of string theory. Then in Appendix A we define some quantities of interest in the theory of Riemann surfaces, in Appendix B we discuss the Schottky parametrization and in Appendix $\mathrm{C}$ we give some details of the sewing technique.

\footnotetext{
${ }^{2}$ As usual, we call $b$-cycles the loops in the worldsheet along the $\tau$ direction and $a$-cycles the spatial loops along $\sigma$.
} 


\section{The sewing technique}

The use of the sewing technique for computing multiloop amplitudes is discussed in detail in [12] for bosonic systems and in [13] for fermionic systems. In order to generalize their results to the twisted case, we will follow closely these two papers and refer to them for the explicit derivation of the results used here as starting point. In this section, we just present the main ideas of the sewing technique in order to clarify its application to the physical/mathematical problem discussed in this paper.

\subsection{The torus}

As anticipated in the introduction, the main idea of the sewing technique is to employ a bootstrap approach and construct $g$-loop amplitudes starting from the tree-level results. The first step is of course the construction of 1-loop amplitudes. This derivation is quite natural and well-known since it is explained in Chapter VIII of Green, Schwarz, and Witten book [18]. In fact, tree-level amplitudes can be written with a special choice for the puncture of the external states putting the first vertex in $z=\infty$ and the last in $z=0$. Thus the first state just describes an outgoing string with $\left\langle s_{1}\right|$ and the last state describes an ingoing string with the ket $\left|s_{N}\right\rangle$. At this point one can simply relax the on-shell condition, insert the propagator $k^{L_{0}}$, and identify the two states

$$
\left\langle 0\left|V_{1}(\infty) V_{2}(1) \ldots V_{N}(0)\right| 0\right\rangle=\left\langle s_{1}\left|V_{2}(1) \ldots V_{N-1}\right| s_{N}\right\rangle \rightarrow \operatorname{Tr}\left[V_{2}(1) \ldots V_{N-1} k^{L_{0}}\right] .
$$

This transforms the v.e.v. of the vertex operators, typical of tree-level amplitudes, into a trace over the Hilbert space of a propagating string; moreover the neighborhood around the points $z=\infty$ and $z=0$ are identified by the projective transformation $\left(z \rightarrow k^{n} z\right)$ generated by the insertion of the propagator. As an example, it is useful to start with the analysis of the vacuum 1-loop amplitude, since it contains some of the features of the general computation and can be derived from the simple trace in (2.1) without external states. In fact, it is not difficult to compute the torus partition function of a fermionic system of spin $(\lambda, 1-\lambda)$ with trivial periodicity conditions on the $a$-cycle and twisted ones along the $b$-cycle. In fact in the sewing construction the $b$-cycles are generated by the identification of the neighborhoods around two points (usually $z=\infty$ and $z=0$ at 1 -loop) enforced by the propagator $P$ (one can take simply $P=k^{L_{0}}$ ). Since we want to have non-trivial periodicity condition along these cycles, we need to deform the propagator by adding an $\epsilon$ dependence so that

$$
b(z)=k^{\lambda} \mathrm{e}^{-2 \pi \mathrm{i} \epsilon}\left(P_{\epsilon}^{-1} b(k z) P_{\epsilon}\right), \quad c(z)=k^{1-\lambda} \mathrm{e}^{2 \pi \mathrm{i} \epsilon}\left(P_{\epsilon}^{-1} c(k z) P_{\epsilon}\right) .
$$

This can be obtained simply by inserting together with the usual propagator $P$ also a factor of $\mathrm{e}^{2 \pi \mathrm{i} \epsilon j_{0}}$, where $j_{0}$ is the fermionic number operator. The torus partition function can be computed as usual just by taking the trace of twisted $P_{\epsilon}=P \mathrm{e}^{2 \pi \mathrm{i} \epsilon j_{0}}$

$$
Z_{\epsilon}^{\lambda}=\operatorname{Tr}\left[k^{L_{0}} \mathrm{e}^{2 \pi \mathrm{i} \epsilon j_{0}}\right]_{\lambda}=\prod_{n=\lambda}^{\infty}\left(\left(1-\mathrm{e}^{2 \pi \mathrm{i} \epsilon} k^{n}\right)\left(1-\mathrm{e}^{-2 \pi \mathrm{i} \epsilon} k^{n}\right)\right) \prod_{r=1-\lambda}^{\lambda-1}\left(1-\mathrm{e}^{2 \pi \mathrm{i} \epsilon} k^{r}\right)
$$


The structure of this result is quite clear: the first two factors come from the action of the modes $c_{-n}$ and $b_{-n}$ with $n \geq \lambda$ respectively, while the last product is related to the special modes $c_{r}$ with $r \in[(1-\lambda),(\lambda-1)]$ only, since the corresponding $b_{r}$ oscillators vanish on the vacuum. If $\lambda$ is integer the result (2.3) can be rewritten as

$$
Z_{\epsilon}^{\lambda \in \mathbb{N}}=(-1)^{\lambda} \mathrm{e}^{\mathrm{i} \pi(2 \lambda-1) \epsilon} k^{\lambda(1-\lambda) / 2} 2 \mathrm{i} \sin \pi \epsilon \prod_{n=1}^{\infty}\left(1-\mathrm{e}^{2 \pi \mathrm{i} \epsilon} k^{n}\right)\left(1-\mathrm{e}^{-2 \pi \mathrm{i} \epsilon} k^{n}\right)
$$

which is clearly vanishing for $\epsilon=0$. This fact has an important geometrical explanation. For integer $\lambda$, there are periodic and regular solutions of the equation of motions for both $b\left(b \Leftrightarrow z^{-\lambda}\right)$ and $c\left(c \Leftrightarrow z^{\lambda-1}\right)$. In fact the singularities in $z=0$ an $z=\infty$ are the fixed points (B.3) of the projective transformation $P=k^{L_{0}}$ and so are outside the fundamental region representing the torus generated by the propagator $P$ (see Appendix $\mathrm{B}$ ). In other words, both $b$ and $c$ have a zero-mode on the torus and thus it is natural that the partition function without any insertion vanishes. For generic fixed points $\xi$ and $\eta$ these zero-modes can be written as

$$
b(z) \Leftrightarrow\left[\frac{\eta-\xi}{(z-\eta)(z-\xi)}\right]^{\lambda}, \quad c(z) \Leftrightarrow\left[\frac{\eta-\xi}{(z-\eta)(z-\xi)}\right]^{1-\lambda} .
$$

The presence of a twist along the $b$-cycles lifts both zero-modes, since the solutions (2.5) do not satisfy the new boundary conditions. This explains why the partition function does not vanish for $\epsilon \neq 0$. Notice that the difference between the number of the $b$ zeromodes and the number of the $c$ zero-modes is always zero for the torus topology, as it should be. In fact the Riemann-Roch theorem ensures that this difference can depend only on the integrals of the curvature and of the gauge field strength. As said before, the twists are equivalent to the presence of a flat connection along the $b$-cycles, and thus the difference between the number of the $b$ and the $c$ zero-modes can not depend on $\epsilon$. To be precise, for the cases we are interested in, the Riemann-Roch theorem says

$$
\# c \text { zero-modes }-\# b \text { zero-modes }=Q(g-1),
$$

where, as usual, the background charge $Q$ is related to the spin $\lambda$ of the fermionic system $(b, c): Q=1-2 \lambda$.

\section{$2.2 \quad$ Higher genus surfaces}

Turning to the study of higher genus surfaces we observe that this procedure, however, can not be straightforwardly generalized, because the vertex operators $V$ in (2.1) describe just the emission of a specific on-shell state. They depend only on the quantum numbers of the emitted string and thus there is no easy way to identify two of them and sum over all intermediate states. In order to this, one should use a generalization [19, 20] of the 
vertex operators, where also the emitted states are described by a whole Hilbert space

$$
\begin{aligned}
& \left\langle\mathcal{V}_{I}^{X}\right|={ }_{I}\left\langle 0, x_{0}=0\right|: \exp \left\{\oint_{0} d z\left(-X^{v}(1-z) \partial_{z} X_{I}(z)\right)\right\}: \\
& \left\langle\mathcal{V}_{I}^{b c}\right|={ }_{I}\langle 0 ; q=-Q|: \exp \left\{\oint_{0} d z\left(b^{v}(1-z) c_{I}(z)-c^{v}(1-z) b_{I}(z)\right)\right\}: .
\end{aligned}
$$

Here the coordinates with the superscript $v$ describe a propagating (virtual) string, while those with a subscript $I$ describe a generic emitted state in the Hilbert space $\mathcal{H}_{I}$. The modes of the two types of fields (anti)-commute among them, since they refer to completely independent states. Let us stress that the bra-vector in Eq. (2.7) is the vacuum in the Hilbert space of the emitted string, so that $\langle\mathcal{V}|$ is an operator in the $v$-Hilbert space of the propagating string. One can think of the vertices $\langle\mathcal{V}|$ as an "off-shell" generalization of the usual bosonic and ghost part (for $Q=-3$ ) of string vertex operators. However, here off-shell does not have the usual meaning as in field theory. On the contrary, offshell just means that the external states have not been specified yet, and thus $\langle\mathcal{V}|$ can be seen as the generator of all possible three string interaction which are obtained by saturating it with physical (on-shell) states.

So far, in the presentation, we always made reference to the space-time interpretation of the formalism typical of string theory. However, the same formalism can be also applied in the context of 2-dimensional free conformal field theory. One has just to consider the two vertices in Eq. (2.7) separately and use them to construct partition functions or Green functions for bosonic or fermionic systems. By using the vertex $\left\langle\mathcal{V}^{b c}\right|$ as ingredient, we can substitute Eq. (2.1) with a new formula that is suited for the generalization of the sewing procedure to higher genus surfaces. Following [13] it is easy to construct the generator of the $N$-point Green function on the sphere $(g=0)$

$$
\left\langle V_{N ; 0}^{b c}\right|={ }_{v}\langle q=0| \prod_{I=1}^{N}\left(\gamma_{I}\left\langle\mathcal{V}_{I}^{b c}\right| \gamma_{I}^{-1}\right)|q=0\rangle_{v},
$$

where the $N$ Hilbert spaces labeled by $I$ describe the external fields and are all independent, while $\gamma_{I}$ are (arbitrary) projective transformations mapping the interaction point from $z=1$ as in (2.7) to the arbitrary point $z_{I}$. Remember that the vertex in (2.8) contains the tensor product of the $N$ vacua ${ }_{I}\langle 0 ; q=-Q|$, but for notational simplicity we write it simply as $\left\langle V_{N ; 0}^{b c}\right|$. Its explicit form is written in Eq. (2.26) of [13] in terms of the representation $E^{\lambda}$ with weight $\lambda$ of the projective group. As we have seen in the previous 1-loop example, the partition function of a fermionic system is in general vanishing because of the presence of zero-modes. For higher genus surfaces, the Riemann-Roch (2.6) theorem shows that this happens also for non-trivial periodicity conditions; so the simplest non-trivial amplitude is the correlation function

$$
Z_{\epsilon}^{\lambda}\left(z_{1}, \ldots, z_{N_{b}}\right)=\left\langle\prod_{I=1}^{N_{b}} b\left(z_{I}\right)\right\rangle_{(\epsilon, \lambda)}=\left\langle V_{N_{b} ; g}^{b c}\right| \prod_{I=1}^{N_{b}}\left(b_{-\lambda}^{(I)}|q=0\rangle_{I}\right) .
$$


where $\left\langle V_{N_{b} ; g}^{b c}\right|$ is the generalization of the vertex (2.8) at genus $g$. Thus $Z_{\epsilon}^{\lambda}$ is the Green function on a surface of genus $g$ with non-trivial boundary conditions along the $b$-cycles and with $N_{b}=|Q|(g-1)$ insertions of the $b$ field. In order to construct $\left\langle V_{N_{b} ; g}^{b c}\right|$ within the sewing approach, we use the generating vertex $\left\langle V_{N ; 0}^{b c}\right|$ with $N=2 g+N_{b}$ and then identify $g$ pairs of Hilbert spaces (labeled by the index $\mu=1, \ldots, 2 g$ ) by means of the twisted propagator that was used also in the torus computation (2.2). Moreover we are interested in the "minimal" correlation function (2.9) that contains only $b$-fields as external states (labeled by the index $\left.I=1, \ldots, N_{b}\right)$. So we can set to zero all the $b^{(I)}$ oscillators in the computation of $\left\langle V_{N_{b} ; g}^{b c}\right|$ and keep only the $c^{(I)}$ fields that will be saturated by the $N_{b}$ $b$-insertions of (2.9). Here we report just the final result for the generating vertex $\left\langle V_{N_{b} ; g}^{b c}\right|$, while the details of the construction are postponed to the Appendix $\mathrm{C}$

$$
\left\langle V_{N_{b}, g}^{b c}\right|=\operatorname{det}(1-H)\left[\prod_{I=1}^{N_{b}}{ }_{I}\langle q=-Q|\right] \mathcal{F}_{N_{b}, g} .
$$

The formal structure of this equation is quite natural. The non-zero mode contribution is given by a (fermionic) Gaussian integral and this is why the determinant $\operatorname{det}(1-H)$ appears. As in the usual case $\epsilon_{\mu}=0$, also for our computation this determinant can be written in terms of the multipliers of the Schottky group

$$
\operatorname{det}(1-H)=\prod_{\alpha}^{\prime} \prod_{n=\lambda}^{\infty}\left(1-\mathrm{e}^{-2 \pi \mathrm{i} \epsilon \cdot N_{\alpha}} k_{\alpha}^{n}\right)\left(1-\mathrm{e}^{2 \pi \mathrm{i} \epsilon \cdot N_{\alpha}} k_{\alpha}^{n}\right) .
$$

$N_{\alpha}$ is a vector with $g$ integer entries; the $\mu^{\text {th }}$ entry counts how many times the Schottky generators $S_{\mu}$ enters in the element of the Schottky group $T_{\alpha}$, whose multiplier is $k_{\alpha}$; the appearance of each $S_{\mu}$ is counted with the exponent sign, so that $S_{\mu}$ contributes 1, while $\left(S_{\mu}\right)^{-1}$ contributes -1 to the global value of $N_{\alpha}^{\mu}$. The product $\prod_{\alpha}^{\prime}$ is over the primary classes of the Schottky group excluding the identity and counting $T_{\alpha}$ and its inverse only once. Eq. (2.11) is the generalization of the first product of (2.3) for an orientable Riemann surface of genus $g$.

The second term of (2.10) takes into account the zero-mode contribution. In this case it is not possible to write a simple expression that is valid for all arbitrary $\lambda$. The complication arises because the zero-mode $b_{s}^{(2 \mu)}$ are entangled with the external oscillators $c_{\lambda}^{(I)}$. Thus it is not possible to derive the zero-mode contribution to the $g$-traces before having computed the scalar product over the $N_{b}$ external Hilbert spaces present in (2.9). This technical problem appears clearly from the formulae of the Appendix C. Thus we now consider directly the correlation functions (2.9). By inserting Eq. (2.10) into (2.9), one gets

$$
Z_{\epsilon}^{\lambda}\left(z_{1}, \ldots, z_{N_{b}}\right) \equiv \operatorname{det}(1-H) \mathcal{F}^{(\lambda)}
$$

where

$$
\mathcal{F}^{(\lambda)}=\left(\prod_{I=1}^{N_{b}}{ }_{I}\langle q=-Q|\right) \mathcal{F}_{N_{b}, g}\left(\prod_{I=1}^{N_{b}} b_{-1}^{(I)}|q=0\rangle_{I}\right)
$$




\section{The case $|Q|=1$}

Let us first focus on the case $Q=-1$, where the conformal weight of $b(z)$ is $\lambda=1$ and the one of $c(z)$ is zero; we consider generic values for the twist $\epsilon_{\mu}$. As explained previously, the non-zero mode contribution is given by (2.11) with $\lambda=1$. On the other hand, in the Appendix C, we show that the contribution of zero-modes contained in $\mathcal{F}$ (see (2.13)), which depends on $g-1$ variables $z_{I}$, is naturally written as a determinant

$$
\mathcal{F}^{(1)}=\operatorname{det}\left(\begin{array}{ccc}
\zeta_{1}\left(z_{1}\right) & \ldots & \zeta_{g}\left(z_{1}\right) \\
\vdots & & \vdots \\
\zeta_{1}\left(z_{g-1}\right) & \ldots & \zeta_{g}\left(z_{g-1}\right) \\
\mathrm{e}^{2 \pi \mathrm{i} \epsilon_{1}}-1 & \ldots & \mathrm{e}^{2 \pi \mathrm{i} \epsilon_{g}}-1
\end{array}\right)
$$

with

$$
\zeta_{\mu}\left(z_{I}\right)=\sum_{\alpha} \mathrm{e}^{2 \pi \mathrm{i}\left(\epsilon \cdot N_{\alpha}+\epsilon_{\mu}\right)}\left[\frac{1}{z_{I}-T_{\alpha} S_{\mu}(0)}-\frac{1}{z_{I}-T_{\alpha}(0)}\right],
$$

where the sum runs over the all the elements of the Schottky group. Thus from Eq. (2.10) one obtains

$$
Z_{\epsilon}^{\lambda=1}\left(z_{1}, \ldots, z_{g-1}\right)=\mathcal{F}^{(1)} \prod_{\alpha}^{\prime} \prod_{n=1}^{\infty}\left(1-\mathrm{e}^{-2 \pi \mathrm{i} \epsilon \cdot N_{\alpha}} k_{\alpha}^{n}\right)\left(1-\mathrm{e}^{2 \pi \mathrm{i} \epsilon \cdot N_{\alpha}} k_{\alpha}^{n}\right) .
$$

The functional form of this result is exactly the expected one: in fact one can interpret the second term with the Schottky product as the non-zero mode contribution to the determinant of the Dirac operator and the first determinant as the zero-mode contribution to the correlation function $Z_{\epsilon}^{\lambda=1}$, written in terms of twisted abelian differentials. However, even if this interpretation will be eventually the correct one, it can not be applied to Eq. (2.16) as it stands. First Eq. (2.15) defines $g$ functions $\zeta$, while, according to the Riemann-Roch (2.6) theorem we have only $g-1$ abelian differentials. Moreover, it is annoying that the origin of the complex plane plays a privileged rôle in (2.15). In particular this means that the $\zeta$ 's just introduced are not regular: in fact, when $T_{\alpha}$ is the identity or $S_{\mu}^{-1}$, they have a pole for $z_{I}=0$, which in general is part of the fundamental domain representing the Riemann surface. It turns out that one can solve these problems simultaneously.

If all the twists $\epsilon_{\mu}$ are trivial, then the determinant is zero because of the last line. This is of course due to the extra zero-mode $(c(z) \Leftrightarrow$ const) appearing in the untwisted case which makes the correlator (2.12) vanish. So let us suppose that at least one $\epsilon$ is non-trivial, for instance $\epsilon_{g} \neq 0$. With this hypothesis it is easy to simplify (2.14) by making a linear combination of each column with the last one so to set to zero the first $g-1$ entries of the last row

$$
\mathcal{F}^{(1)}=\operatorname{det}\left(\begin{array}{cccc}
\Omega_{1}\left(z_{1}\right) & \ldots & \Omega_{g-1}\left(z_{1}\right) & \zeta_{g}\left(z_{1}\right) \\
\vdots & & \vdots & \vdots \\
\Omega_{1}\left(z_{g-1}\right) & \ldots & \Omega_{g-1}\left(z_{g-1}\right) & \zeta_{g}\left(z_{g-1}\right) \\
0 & \ldots & 0 & \mathrm{e}^{2 \pi \epsilon_{g}}-1
\end{array}\right),
$$


where

$$
\Omega_{\mu}\left(z_{I}\right)=\zeta_{\mu}\left(z_{I}\right)-\frac{\mathrm{e}^{2 \pi \mathrm{i} \epsilon_{\mu}}-1}{\mathrm{e}^{2 \pi \mathrm{i} \epsilon_{g}}-1} \zeta_{g}\left(z_{I}\right), \quad \mu=1, \ldots, g-1 .
$$

This shows that only the $g-1$ functions $\Omega$ enter in the final result (2.16) so that it is natural to identify these $\Omega$ 's with the twisted abelian differentials. Let us shows that they have all the expected properties. First, the dependence on the origin disappeared and, contrary to the original $\zeta$ 's, the $\Omega$ 's are everywhere regular. To see this, it is useful to rewrite the sum $(2.15)$ in a different form ${ }^{3}$, separating the contributions coming from the Schottky elements of the form $T_{\alpha} S_{\mu}^{l}$

$$
\begin{aligned}
\zeta_{\mu}\left(z_{I}\right) & =\sum_{\alpha}^{(\mu)} \mathrm{e}^{2 \pi \mathrm{i}\left(\epsilon \cdot N_{\alpha}+\epsilon_{\mu}\right)}\left[\frac{1}{z_{I}-T_{\alpha}\left(\eta_{\mu}\right)}-\frac{1}{z_{I}-T_{\alpha}\left(\xi_{\mu}\right)}\right] \\
& +\left(1-\mathrm{e}^{2 \pi \mathrm{i} \epsilon_{\mu}}\right) \sum_{\alpha} \mathrm{e}^{2 \pi \mathrm{i} \epsilon \cdot N_{\alpha}}\left[\frac{1}{z_{I}-T_{\alpha}(0)}-\frac{1}{z_{I}-T_{\alpha}\left(a_{\mu}^{\alpha}\right)}\right],
\end{aligned}
$$

where, in the second line, $a_{\mu}^{\alpha}=\eta_{\mu}$ if the $T_{\alpha}$ is of the form $T_{\alpha}=T_{\beta} S_{\mu}^{l}$ with $l \geq 1$, while $a_{\mu}^{\alpha}=\xi_{\mu}$ otherwise. It is also important to remember that the sum in the first line does not contain all the Schottky elements whose rightmost generator is $S_{\mu}^{ \pm 1}$, while the second sum is over all the elements $T_{\alpha}$. It is now easy to see that first term in the second square bracket of (2.19) cancels in the combination (2.18). Then one can check the periodicity properties. It is clear that all the expressions we have written so far are periodic along the $a$-cycles $(z-\eta) \rightarrow \mathrm{e}^{2 \pi \mathrm{i}}(z-\eta)$, since they are holomorphic in $z$. The periodicity along the $b$-cycles can be deduced by looking at the Eq. (2.15). By means of the identity (B.8) one can see that

$$
\zeta_{\mu}\left(S_{\nu}(z)\right) d S_{\nu}(z)=\mathrm{e}^{2 \pi \mathrm{i} \epsilon_{\nu}} \zeta_{\mu}(z) d z
$$

Since the $\Omega$ 's are simply linear combinations of the $\zeta$ 's, they have the same periodicity property, which is exactly what one expected from Eq. (2.2). The fermionic result (2.16) is multilinear in the abelian differentials $\Omega$ 's and thus has a non-trivial periodicity along the $b$-cycles $(2.20)$

$$
Z_{\epsilon}^{\lambda=1}\left(z_{1}, \ldots, S_{\nu}\left(z_{k}\right), \ldots, z_{g-1}\right) S_{\nu}^{\prime}\left(z_{k}\right)=\mathrm{e}^{2 \pi \mathrm{i} \epsilon_{\nu}} Z_{\epsilon}^{\lambda=1}\left(z_{1}, \ldots, z_{g-1}\right) .
$$

Notice that, if some $\epsilon_{\mu}=0$ (of course with $\mu \neq g$ ), the corresponding $\Omega_{\mu}$ reduces to $\zeta_{\mu}$, where the second line of (2.19) vanishes. This is the naïve generalization of the usual untwisted abelian differentials $\omega_{\mu}\left(z_{I}\right)$ (given in (B.6)) with an additional phase $\exp \left(2 \pi \mathrm{i} \epsilon \cdot N_{\alpha}\right)$, which ensures the twisting of the periodicity along the cycles $b_{\nu}$ with $\nu \neq \mu$. For the general case $\epsilon_{\mu} \neq 0$, the twisted periodicity along $b_{\mu}$ requires also the second line of Eq. (2.19).

\footnotetext{
${ }^{3}$ See the Appendix $\mathrm{C}$ for the derivation.
} 


\section{The case $|Q| \geq 2$}

The generalization of the result just presented to the case $\lambda=3 / 2,2, \ldots$ is discussed in Appendix C. We recall here the main qualitative features. $\mathcal{F}^{(\lambda)}(\mathrm{C} .17)$ has the same structure of $\mathcal{F}^{(1)}$, but the elements of this new matrix are themselves $(2 \lambda-1) \times(2 \lambda-1)$ blocks. This is simply because the zero-modes now are related to the oscillators $b_{r}$, with $r \in[(1-\lambda),(\lambda-1)]$. The presence of many zero-modes implies that, in the "minimal" correlation function $(2.9)$ there are $(2 \lambda-1)(g-1)$ variables $z_{I}$ and that the building blocks of the matrix $\mathcal{F}^{(\lambda)}$ have extra indices $r, s \in[(1-\lambda),(\lambda-1)]$ and become $\zeta_{\mu, s}\left(z_{\nu, r}\right)$. Also the entries of the last row in $\mathcal{F}^{(\lambda)}$ are now replaced by $(2 \lambda-1) \times(2 \lambda-1)$ matrices

$$
\mathcal{E}\left(S_{\mu}\right)_{r s}=\left(\mathrm{e}^{2 \pi \mathrm{i} \epsilon_{\mu}} E\left(S_{\mu}\right)-11\right)_{r s} .
$$

The $\zeta_{\mu, s}\left(z_{\nu, r}\right)$ 's suffer the same diseases discussed in the $\lambda=1$ case. These problems are cured in a similar way by defining $(2 \lambda-1)(g-1) \lambda$-differentials $\Omega_{\mu, s}\left(z_{\nu, r}\right)$, analogous to the ones introduced in (2.18) (see Eq. (C.22)). An important difference with the respect to the $\lambda=1$ case is that, for $\lambda>1$ and $g>1$, the determinant $\mathcal{F}^{(\lambda)}$ does not vanish even if $\epsilon_{\mu}=0, \forall \mu$. Therefore the correlation function (2.12) is non-trivial also when all the $\epsilon_{\mu}$ are set to zero. Notice that, if $\lambda$ is half-integer, then the expression (C.22) can be used also in absence of twists $(\epsilon=0)$. In fact in this case the matrix $\mathcal{E}\left(S_{\mu}\right)_{r s}$ is always invertible. For integer $\lambda$, on the contrary, the determinant of $\mathcal{E}\left(S_{\mu}\right)_{r s}$ is vanishing for $\epsilon=0$ and the form of the $\lambda$-differentials is more complicated, as discussed in [13]. Of course one can still express all the correlation function in terms of the $\zeta_{\mu, s}\left(z_{\nu, r}\right)$ introduced in (C.18).

\section{Comparison with bosonic determinants}

We are now in the position to generalize Eq. (1.1). As anticipated in the Introduction, this kind of relations can be viewed as a consequence of the equivalence between fermionic and bosonic systems. In fact, using again the sewing technique, it is possible to derive the bosonic correlation functions corresponding to $Z_{\epsilon}^{\lambda}$ of Eq. (2.9). In the notation of [12] these correlators are

$$
Z_{\epsilon_{b}}^{Q}\left(z_{1}, \ldots, z_{N_{b}}\right)=\left\langle\prod_{I=1}^{N_{b}}: \mathrm{e}^{-\phi\left(z_{I}\right)}:\right\rangle_{\left(\epsilon_{b}, Q\right)}=\left\langle V_{N_{b} ; g}^{\phi}\right| \prod_{I=1}^{N_{b}}\left(|q=-1\rangle_{I}\right),
$$

where the bosonic system has background charge $Q=1-2 \lambda$. Moreover the twisted boundary conditions along the $b$-cycles are enforced on the bosonic side by the insertion together with the propagator of the operator $\mathrm{e}^{2 \pi \mathrm{i} \epsilon_{b} p_{0}}[12]$. Notice, however, that $\epsilon_{b}=$ $\epsilon-1 / 2$, where the additional factor of $1 / 2$ is necessary in order to reproduce in the bosonic language the usual $(-1)^{F}$-twist of the fermionic traces (see for instance Appendix A of [21], Eq. (A.2.23)).

In order to provide a simple example, let us write the 1-loop partition function of a bosonic system with $Q=1-2 \lambda$ with twist $\epsilon_{b}$ (see, for instance, Eq. (3.21) of [12] with 
$\alpha=0, \beta=\epsilon_{b}$ and $\left.N_{1}=N_{2}=0\right)$

$$
Z_{\epsilon_{b}}=\prod_{n=1}^{\infty}\left(1-k^{n}\right)^{-1} \theta\left(\epsilon_{b}-Q\left(\frac{1}{2}+\Delta\right) \mid \tau\right)
$$

This formula has to be equal to Eq. (2.3) for $\epsilon_{b}=\epsilon-1 / 2$ and from this relation one can derive the usual product formula for the 1-loop Theta function. In particular, in terms of the odd $\theta_{1}$, one has

$$
\theta_{1}(\epsilon \mid \tau)=2 k^{1 / 8} \sin \pi \epsilon \prod_{n=1}^{\infty}\left(1-k^{n}\right)\left(1-\mathrm{e}^{2 \pi \mathrm{i} \epsilon} k^{n}\right)\left(1-\mathrm{e}^{-2 \pi \mathrm{i} \epsilon} k^{n}\right)
$$

The higher genus case works in a similar way. The main difference is that the partition function without insertions now vanishes. On the fermionic side we know that this is due to the presence of zero-modes, while on the bosonic side this is a simple consequence of the momentum conservation modified by the background charge. Thus, in order to obtain a non-trivial result, we have to compare the correlation functions (2.12) and (3.1). For $Q=-1$ the fermionic result has been obtained in Section 4 and the bosonic one ${ }^{4}$ is (again from Eq. (3.21) of [12])

$$
Z_{\epsilon_{b}}^{Q=-1}\left(z_{1}, \ldots, z_{g-1}\right)=\frac{\prod_{I=1}^{g-1} \sigma\left(z_{I}\right) \prod_{I<J} E\left(z_{I}, z_{J}\right)}{\prod_{\alpha}^{\prime} \prod_{n=1}^{\infty}\left(1-k_{\alpha}^{n}\right)} \theta\left(\epsilon+\Delta-\sum_{I=1}^{g-1} J\left(z_{I}\right) \mid \tau\right)
$$

where we have used again $\epsilon_{b \mu}=\epsilon_{\mu}-1 / 2 \forall \mu=1, \ldots, g$. Notice that, thanks to (A.2) and (A.6), the argument of the Theta-function on the r.h.s. does not depend on the basis point $z_{0}$ of the Jacobi map. The explicit expressions in terms of the Schottky parametrization of $\sigma\left(z_{I}\right)$ (including its normalization), $E\left(z_{I}, z_{J}\right)$ and $\Delta$ are given in Appendix A of [12].

The equivalence with the fermionic result implies ${ }^{5}(2.16)=(3.4)$

$$
C_{\epsilon}^{(1)} \mathcal{F}^{(1)}=\prod_{I=1}^{g-1} \sigma\left(z_{I}\right) \prod_{I<J} E\left(z_{I}, z_{J}\right) \theta\left(\epsilon+\Delta-\sum_{I=1}^{g-1} J\left(z_{I}\right) \mid \tau\right)
$$

which generalizes (1.1) to the twisted case, with

$$
C_{\epsilon}^{(1)}=\prod_{\alpha}^{\prime} \prod_{n=1}^{\infty}\left(1-k_{\alpha}^{n}\right)\left(1-\mathrm{e}^{-2 \pi \mathrm{i} \epsilon \cdot N_{\alpha}} k_{\alpha}^{n}\right)\left(1-\mathrm{e}^{2 \pi \mathrm{i} \epsilon \cdot N_{\alpha}} k_{\alpha}^{n}\right) .
$$

A first check of this identity comes from the study of the periodicity properties of the results. The periodicity (2.21) is reproduced in the bosonic side in a complicated way

\footnotetext{
${ }^{4}$ In Appendix A we recall the definition and the main properties of the various geometrical objects present in (3.4).

${ }^{5}$ From now on, we endow the $\lambda$-differentials derived in the sewing approach with the appropriate factors of $d z^{\lambda}$.
} 
by combining the various factors coming from transformation of the objects on the r.h.s. of (3.4). However most of these contributions cancel as in the untwisted case and the only $\epsilon$-dependent contribution comes from the Theta function. From Eqs. (A.4), (A.8) and (A.9), one gets

$$
Z_{\epsilon_{b}}^{Q=-1}\left(z_{1}, \ldots, S_{\nu}\left(z_{k}\right), \ldots, z_{g-1}\right) S_{\nu}^{\prime}\left(z_{k}\right)=\mathrm{e}^{2 \pi \mathrm{i} \epsilon_{\nu}} Z_{\epsilon_{b}}^{Q=-1}\left(z_{1}, \ldots, z_{g-1}\right)
$$

in agreement with (2.21). Actually also a stronger check of (3.5) is possible. Since we know the explicit form for the twisted abelian differentials (2.18), we can use it together with the expressions contained in Appendix A of [12] in order to express both sides of (3.5) in terms of the Schottky group. At this point one can develop the results for small $k_{\mu}$ and keep only a finite number of terms in all the series or infinite products so to obtain in both sides a polynomial in the $k_{\mu}$ 's. The coefficients of each term are (complicated) functions of the fixed points and of the twisting parameters $\epsilon$ 's. A highly non-trivial check is to verify that the coefficients obtained on the l.h.s. of (3.5) agree with those obtained from the expansion of the r.h.s. We performed this check for the case $g=2$ and verified that the first four terms in the expansion of (3.5) actually agree.

The generalization of (3.5) to the case $|Q| \geq 2$ is straightforward and reads 6

$$
C_{\epsilon}^{(\lambda)} \mathcal{F}^{(\lambda)}=\frac{\prod_{I<J} E\left(z_{I}, z_{J}\right)}{\prod_{I=1}^{N_{b}} \sigma\left(z_{I}\right)^{Q}} \theta\left(\epsilon-\frac{1}{2}-Q\left(\Delta+\frac{1}{2}\right)-\sum_{I=1}^{N_{b}} J\left(z_{I}\right) \mid \tau\right)
$$

with

$$
C_{\epsilon}^{(\lambda)}=\prod_{\alpha}^{\prime}\left(\prod_{n=1}^{\infty}\left(1-k_{\alpha}^{n}\right) \prod_{n=\lambda}^{\infty}\left(1-\mathrm{e}^{-2 \pi \mathrm{i} \epsilon \cdot N_{\alpha}} k_{\alpha}^{n}\right)\left(1-\mathrm{e}^{2 \pi \mathrm{i} \epsilon \cdot N_{\alpha}} k_{\alpha}^{n}\right)\right)
$$

\section{Conclusions}

In this paper we exploited the sewing technique to study free fermions of arbitrary spin (and free boson with arbitrary background charge) with twisted boundary conditions on the $b$-cycles. We computed various quantities of interest like determinants and twisted differentials and gave explicit expressions for them in terms of the Schottky uniformization of the Riemann surface. By comparing the bosonic and the fermionic results for certain "minimal" correlation functions we also found new identities between products of multipliers over the Schottky group and the usual multiloop Theta functions. Identities of this kind are important because they make manifest the properties under modular transformations which are hidden when the results are written in Schottky parametrization. This is very useful, for instance, in string theory, where the modular properties of perturbative amplitudes are crucial for the consistency of the theory. On the other hand, important building blocks of string amplitudes, like the partition functions, are often given in terms of Schottky product like (3.6) and thus their modular properties are

\footnotetext{
${ }^{6}$ With a small abuse of notation, the term of $1 / 2$ in (3.8) stays for a $g$-component vector whose entries are all $1 / 2$.
} 
blurred. It is clearly important to rewrite these products in terms of Theta-functions. Let us mention here an example of this problem in the context of open bosonic strings [22].

It is well known that a system of $g+1$ D-branes, connected among them with tubes representing the closed string propagation, is equivalently described by a disk with $g$ holes. The two descriptions make manifest different unitarity properties of the result: in the D-brane picture the poles of the amplitude in the Schottky multipliers are related to the propagation of some almost on-shell closed string states between D-branes, while in the disk description the poles are related to open string states. The difference is made more evident if one switches on a constant gauge field strength $F$ on the D-branes (or, in the $T$-dual language, if some of the D-branes in this multi-body system have a non-vanishing constant relative velocity). In the D-brane language the result can be derived by introducing in the construction of [23] a twist similar to the one discussed in this paper. In this case $\epsilon$ is related to the differences between the external fields $F$. In the D-brane description these twists enter only as a phase independent of the moduli, exactly as in (3.6). By using the results of this paper one can explicitly perform the modular transformation of this expression and obtain the same quantity in the open string description, where the surface looks like a disk with $g$ holes. Now the phases induced by the twists do depend on the moduli and this is strictly related to the modification induced by $F$ on the mass of the open strings. This very important difference is already present at 1-loop level (i.e. in a system of two D-branes), as it is clear by the comparison between the results of [24] and [25]. We have checked [22] that, as already suggested in [24], from the open string formulation of the charged partition function one can derive the 1-loop Euler-Heisenberg effective action for Yang-Mills theories simply by performing the field theory limit $\alpha^{\prime} \rightarrow 0$ of the results, in the spirit of [26] (see also references therein). Of course one obtains the pure Yang-Mills effective action if the bosonic D3-branes are used as starting point, or the $\mathcal{N}=4$ result if one starts from the usual type IIB D3-branes. This technique can be extended to more complicated systems of $\mathcal{N}=2$ theories with various content of matter [27], once the corresponding string results are derived. On the contrary, the description of [25] and its bosonic multiloop version [23] are not directly connected to the Yang-Mills effective action. In this case the low energy limit captures the (super)gravity interaction among the D-branes seen as classical gravitational solitons. Notable exceptions to this are known, where perturbative gauge theory results are directly connected to supergravity computations. This striking connection is usually due to the cancellation of the contribution related to the stringy modes. In fact, if the complete string result is due only to massless modes exchange (of the closed and the open strings respectively in the two descriptions), then the usual open/closed string duality connects directly gauge theory quantity to supergravity tree-diagrams [28]. Of course this does not happen for the whole effective action even in the maximally supersymmetric models, but it is a possibile feature of only the first non-vanishing term in the small $F$ (or small $\epsilon)$ expansion.

Coming back to the technical construction presented in this paper, the main open problem is to extend the sewing approach to the case where one has twists along the $a$-cycles. As just argued, one can avoid this problem for certain cases by exploiting the 
modular properties of the results. However, modular transformations cannot be enough when twists are present both along the $a$-cycles and the $b$-cycles at the same time. This seems a challenging problem, since even in the simplest situations, one looses the usual relation between string amplitudes and the Schottky group. The main difficulty is that the building blocks of the amplitudes have not been expressed so far in terms of representation of the projective group, see [17].

\section{Acknowledgments}

We would like to thank C.S. Chu for collaboration on related topics and for interesting discussions and suggestions on the results presented here. We would like to thank Andrew McIntyre for email exchange and for pointing out to us various interesting papers in the mathematical literature. This work is supported in part by EU RTN contract HPRNCT-2000-00131 and by MIUR contract 2001-025249. R.R. is supported by European Commission Marie Curie Postdoctoral Fellowships.

\section{Appendix A: Definitions}

In this Appendix, we collect the definitions of some quantities of relevant interest in the theory of Riemann surfaces.

Let $\Sigma$ be a compact Riemann surface of genus $g$ with a complex structure defined on it. There exists a normalized basis of holomorphic 1 -forms $\omega_{\mu}(\mu=1, \ldots, g)$, called abelian differentials, such that

$$
\frac{1}{2 \pi \mathrm{i}} \oint_{a_{\mu}} \omega_{\nu}=\delta_{\mu \nu} \quad, \quad \frac{1}{2 \pi \mathrm{i}} \oint_{b_{\mu}} \omega_{\nu}=\tau_{\mu \nu},
$$

where $a_{\mu}$ and $b_{\mu}$ are a canonical basis of homology cycles with intersection matrix: $I\left(a_{\mu}, b_{\nu}\right)=\delta_{\mu \nu}, I\left(a_{\mu}, a_{\nu}\right)=I\left(b_{\mu}, b_{\nu}\right)=0$. The matrix $\tau$ is called period matrix on $\Sigma$ and is a symmetric matrix with positive definite imaginary part.

Given a base point $z_{0} \in \Sigma$, one can associate to any $z \in \Sigma$ a complex $g$-component vector $J(z)$ by means of the Jacobi map

$$
J: z \rightarrow J_{\mu}(z)=\frac{1}{2 \pi \mathrm{i}} \int_{z_{0}}^{z} \omega_{\mu} .
$$

The vector $J$ is defined up to periods around $a_{\mu}$ or $b_{\mu}$ (A.1) and so belongs to the complex torus $J(\Sigma)=\mathbb{C}^{g} /\left(\mathbb{Z}^{g}+\tau \mathbb{Z}^{g}\right)$ called Jacobi variety.

The Riemann Theta function associated to the surface $\Sigma$ is defined for $Z \in \mathbb{C}^{g}$ by

$$
\theta(Z \mid \tau)=\sum_{n \in \mathbb{Z}^{g}} \exp \{\mathrm{i} \pi n \cdot \tau \cdot n+2 \pi \mathrm{i} n \cdot Z\}
$$


where $n \cdot \tau \cdot n=\sum_{\mu, \nu=1}^{g} n_{\mu} \tau_{\mu \nu} n_{\nu}$ and $n \cdot Z=\sum_{\mu=1}^{g} n_{\mu} Z_{\mu}$. The Theta function has a simple transformation law under shifts of $Z$ on the lattice $\mathbb{Z}^{g}+\tau \mathbb{Z}^{g}$ :

$$
\theta(Z+\tau \cdot n+m \mid \tau)=\exp [-\pi \mathrm{i} n \cdot \tau \cdot n-2 \pi \mathrm{i} n \cdot Z] \theta(Z \mid \tau)
$$

The Riemann vanishing theorem states that $\theta(Z \mid \tau)$ vanishes if and only if there exist $g-1$ points $\left(z_{1}, \ldots, z_{g-1}\right)$ on $\Sigma$ such that

$$
Z=\Delta_{\left(z_{0}\right)}-\sum_{\rho=1}^{g-1} J\left(z_{\rho}\right)
$$

where $\Delta_{\left(z_{0}\right)}$ is a constant vector in $J(\Sigma)$ (called Riemann class) and the vectors $J\left(z_{\rho}\right)$ are defined by (A.2). The dependence of $\Delta_{\left(z_{0}\right)}$ from the base point $z_{0}$ is given by:

$$
\Delta_{(z)}=\Delta_{\left(z_{0}\right)}-\frac{g-1}{2 \pi \mathrm{i}} \int_{z_{0}}^{z} \omega
$$

We also used two other important functions defined on $\Sigma: E(z, y)$ and $\sigma(z)$.

The prime form $E(z, y)$ is completely defined by the following properties:

- it is a holomorphic differential form on $\Sigma \otimes \Sigma$ of weight $(-1 / 2,0) \times(-1 / 2,0)$;

- it is odd under the exchange $z \leftrightarrow w$;

- it has a simple zero for $z \rightarrow w$ with the normalization

$$
E(z, w) \underset{z \rightarrow w}{\longrightarrow} \frac{z-w}{\sqrt{d z} \sqrt{d w}}
$$

- it is single valued around the $a$-cycles, but not around the $b$-cycles: if $z$ goes around $b_{\mu}$ the prime form is shifted in the following way

$$
E(z, w) \longrightarrow-\exp \left[-\pi \mathrm{i} \tau_{\mu \mu}-\int_{w}^{z} \omega_{\mu}\right] E(z, w)
$$

The weight $(-1 / 2,0) \times(-1 / 2,0)$ means that $E(z, w)$ contains the square root of the differentials $d z, d w$ at the denominator. The rôle of these differentials becomes clear on the sphere, where $E(z, w)$ is exactly given by the r.h.s. of Eq. (A.7). In fact the differentials make $E(z, w)$ invariant under inversion: $z \rightarrow 1 / z, w \rightarrow 1 / w$ and therefore assure a regular behaviour of the prime form at the infinity.

Finally the function $\sigma(z)$ is defined up to a constant factor by the following properties

- it is a holomorphic differential form on $\Sigma$ of weight $g / 2$,

- it has no zeros, 
- it is single valued around the $a$-cycles and transforms as

$$
\sigma(z) \longrightarrow \exp \left[\pi \mathrm{i}(g-1) \tau_{\mu \mu}+\pi \mathrm{i}-2 \pi \mathrm{i}\left(\Delta_{z}\right)_{\mu}\right] \sigma(z)
$$

when $z$ is moved around the cycle $b_{\mu}$.

In the second part of this Appendix we will discuss the properties of these quantities under the modular transformations, that is under a change of the canonical homology basis. Two homology basis $(a, b)$ and $(\tilde{a}, \tilde{b})$ are related by a modular transformation if there is a $2 g \times 2 g$ matrix with integer entries

$$
\left(\begin{array}{ll}
A & B \\
C & D
\end{array}\right) \in S p(2 g, \mathbb{Z})
$$

such that

$$
\left(\begin{array}{c}
\tilde{b} \\
\tilde{a}
\end{array}\right)=\left(\begin{array}{ll}
A & B \\
C & D
\end{array}\right)\left(\begin{array}{l}
b \\
a
\end{array}\right)
$$

The $g \times g$ blocks of the matrix in (A.10) satisfy the constraints $A B^{t}=B A^{t}, C D^{t}=D C^{t}$, $A D^{t}-B C^{t}=1_{g}$, which assure the invariance of the intersection matrix ${ }^{7}$. In order to preserve the canonical normalization of the abelian differentials (A.1), the $\tilde{\omega}$ in the new basis are related to the old ones by

$$
\tilde{\omega}=\omega \cdot(C \tau+D)^{-1}
$$

and correspondingly one gets the new period matrix $\tilde{\tau}$

$$
\tilde{\tau}_{\mu \nu}=\frac{1}{2 \pi \mathrm{i}} \oint_{\tilde{b}_{\mu}} \tilde{\omega}_{\nu}=\left[(A \tau+B)(C \tau+D)^{-1}\right]_{\mu \nu}
$$

The transformation of the prime form under (A.11) is given, for example, in [11]

$$
\tilde{E}(z, w)=\exp \left[\frac{1}{4 \mathrm{i} \pi} \int_{z}^{w} \omega \cdot(C \tau+D)^{-1} C \cdot \int_{z}^{w} \omega\right] E(z, w),
$$

The transformation properties of the Riemann class (A.6), the Riemann Theta function (A.3) and the functions $\sigma$ depend on the values of the diagonal elements of $C D^{t}$ and $A B^{t}$. Here we focus on the case where $\left(C D^{t}\right)_{\mu \mu}=\left(A B^{t}\right)_{\mu \mu}=2 \mathbb{Z}, \forall \mu=1, \ldots, g$. The Riemann class (A.6) has a simple transformation property under these elements of the modular group

$$
\tilde{\Delta}=\Delta(C \tau+D)^{-1}
$$

The Riemann Theta function (A.3) transforms as

$$
\theta(\tilde{Z} \mid \tilde{\tau})=\xi \sqrt{\operatorname{det}(C \tau+D)} \mathrm{e}^{\mathrm{i} \pi Z \cdot(C \tau+D)^{-1} C \cdot Z} \theta(Z \mid \tau)
$$

\footnotetext{
${ }^{7}$ Notice that the square of the intersection matrix is -1 , which implies that also the transpose of the matrix in (A.10) belongs to $S p(2 g, \mathbb{Z})$; so we also have $A^{t} C=C^{t} A, B^{t} D=D^{t} B, A^{t} D-C^{t} B=1_{g}$.
} 
where $\tilde{Z}=Z(C \tau+D)^{-1}$ and $\xi$ is a phase such that $\xi^{8}=1$ depending only on the modular transformation. Finally the function $\sigma$ undergoes the following transformation:

$$
\tilde{\sigma}(z)=K \exp \left[-\frac{\mathrm{i} \pi}{g-1} \Delta_{z} \cdot(C \tau+D)^{-1} C \cdot \Delta_{z}\right] \sigma(z),
$$

where $K$ is a normalization factor independent of $z$.

\section{Appendix B: The Schottky parametrization}

It is well known that one can represent the sphere through the stereographic projection as the extended complex plane: $\mathbb{C} \cup\{\infty\}$. This equivalence is the starting point to represent any Riemann surface as part of the extended complex plane by means of the so-called Schottky uniformization. Here we will focus on closed orientable surfaces and will give a concrete realization of the intuitive idea that one can generate higher genus surfaces just by adding handles to the sphere. As an example let us derive the Schottky parametrization of the torus. It is sufficient to perform two operations. First one has to choose in $\mathbb{C}$ two points $J=-d / c$ and $J^{\prime}=a / c$ and cut around them two non-overlapping disks $\mathcal{C}$ and $\mathcal{C}^{\prime}$ of the same radius $R=1 /|c|$. Then one has to identify the two circles, which are the borders of the disks, so to obtain again a closed surface (actually this identification can be done with a twist of an arbitrary angle $\theta$ related to the phase of $c$ ). These two operations can be summarized mathematically by introducing the loxodromic projective transformation $S \in S L(2, \mathbb{C})$

$$
S=\left(\begin{array}{ll}
a & b \\
c & d
\end{array}\right) \quad, \quad \text { with } a d-b c=1 \quad \text { and } \quad(\operatorname{Tr} S)^{2} \notin[0,4] \text {. }
$$

This defining transformation is called generator, while the complete Schottky group $\mathcal{S}$ is obtained by all the possible products of the $S^{\text {' }}$ s (just $S^{n}$ with $n \in \mathbb{Z}$ in the case of the torus). The isometric circles are described by the equations:

$$
\left|\frac{d S}{d z}\right|^{-1 / 2}=|c z+d|=1, \quad\left|\frac{d S^{-1}}{d z}\right|^{-1 / 2}=|c z-a|=1,
$$

in agreement with what we have said above. The requirement of having two nonoverlapping disks ensures that the projective map $S$ introduced in (B.1) is loxodromic. This kind of transformation can be equivalently characterized by a couple of fixed points $(\eta, \xi)$

$$
\lim _{n \rightarrow \infty} S^{n}(z)=\eta, \quad \lim _{n \rightarrow \infty} S^{-n}(z)=\xi, \quad \forall z \neq \xi, \eta
$$

and by a multiplier $k$

$$
k=\frac{S(z)-\eta}{S(z)-\xi} \frac{z-\xi}{z-\eta}, \quad \text { with }|k|<1
$$


valid $\forall z \neq \xi, \eta$. A generic loxodromic transformation $S$ can be written in terms of its fixed points and multiplier in a simple way

$$
S=\frac{1}{\sqrt{k}(\xi-\eta)}\left(\begin{array}{cc}
\eta-k \xi & -\xi \eta(1-k) \\
1-k & k \eta-\xi
\end{array}\right) .
$$

Notice that $S$ maps any point outside the circle $\mathcal{C}$ into a point inside the circle $\mathcal{C}^{\prime}$. On the contrary $S^{-1}$ maps any point outside the circle $\mathcal{C}^{\prime}$ into a point inside the circle $\mathcal{C}$. Thus the fundamental region representing the torus is simply the extended complex plane minus the fixed points, modulo the equivalence relation induced by the various elements of $\mathcal{S}:(\mathbb{C} \cup\{\infty\}-\{\xi, \eta\}) / \mathcal{S}$.

In general, one can apply these same ideas to build a surface of genus $g$ : it is sufficient to cut in the extended complex plane $g$ pairs of non-overlapping disks and identify the pairs of the corresponding circles. Clearly this corresponds to the insertion of $g$ handles on the sphere. Mathematically this construction is described by $g$ loxodromic projective transformations $S_{\mu}$ that generate freely the Schottky group $\mathcal{S}_{g}$. As described in the Appendix $\mathrm{C}$, in the sewing procedure the generators of the group naturally arise from combinations of the local coordinates and of the propagator (see in particular Eq. (C.7)). So a Riemann surface with $g$ holes is represented by the extended complex plane, minus all the fixed points, modded out by the equivalence relation induced by $\mathcal{S}_{g}$. In this uniformization of the surface the $a$-cycles correspond to the circle $\mathcal{C}^{\prime}$ anti-clockwise oriented, while the $b$-cycles are represented by lines connecting $z$ and $S_{\mu}(z)$ Notice that the condition of having non-overlapping circles ensures that each element $T_{\alpha}$ of $\mathcal{S}_{g}$ different from the identity is a loxodromic map and so can be characterized by the fixed points $\left(\eta_{\alpha}, \xi_{\alpha}\right)$ and by the multiplier $k_{\alpha}$. In the text we also make use of the following standard nomenclature:

- A primitive element in the group is a transformation which can not be written as an integer power of any other element;

- A conjugacy class is the set of the elements that can be related to each other by a cyclic permutation of their constituent factors (for instance, $S_{1} S_{2}$ and $S_{2} S_{1}$ belong to the same conjugacy class);

- A primary class is a conjugacy class containing only primitive elements.

All the quantities living on Riemann surfaces introduced in an axiomatic way in thep revious appendix can be explicitly written in terms of Poincaré series on the elements of the Schottky group (see Appendix A of [12]). We report here only the abelian differentials for a surface of genus $g$

$$
\omega_{\mu}(z)=\sum_{\alpha}^{(\mu)}\left(\frac{1}{z-T_{\alpha}\left(\eta_{\mu}\right)}-\frac{1}{z-T_{\alpha}\left(\xi_{\mu}\right)}\right) d z \quad, \quad \mu=1, \ldots, g .
$$

where $\sum_{\alpha}{ }^{(\mu)}$ means that the sum is over all the elements of the Schottky group that do not have $S_{\mu}^{n}, n \in \mathbb{Z}-\{0\}$, as their rightmost factor and $\eta_{\mu}, \xi_{\mu}$ are the fixed points of the generator $S_{\mu}$. 
Finally it is very useful to notice the following identity

$$
\frac{T(a)-T(b)}{T(a)-T(d)} \frac{T(c)-T(d)}{T(c)-T(b)}=\frac{a-b}{a-d} \frac{c-d}{c-b}
$$

valid for any projective transformation $T$ and for any points $a, b, c$ and $d$. This means that the cross ration of (B.7) is invariant under projective transformation. For instance, this identity can be used to rewrite the combinations that typically appear in the expressions for the abelian differentials

$$
\begin{aligned}
T^{\prime}(z)\left(\frac{1}{T(z)-x}-\frac{1}{T(z)-y}\right) & =\frac{d}{d z} \log \left(\frac{T(z)-x}{T(z)-y} \frac{T\left(z_{0}\right)-y}{T\left(z_{0}\right)-x}\right) \\
& =\frac{1}{z-T^{-1}(x)}-\frac{1}{z-T^{-1}(y)} .
\end{aligned}
$$

For $T=S_{\nu}, x=T_{\alpha}\left(\eta_{\mu}\right)$ and $y=T_{\alpha}\left(\xi_{\mu}\right)$, Eq. (B.8) shows that $\omega_{\mu}$ is periodic when $z$ goes around a cycle $b_{\nu}$ (i.e. $\left.z \rightarrow S_{\nu}(z)\right)$.

\section{Appendix C: The twisted sewing}

For trivial boundary conditions $\epsilon_{\mu}=0$, the derivation of the generating vertex $\left\langle V_{N_{b} ; g}^{b c}\right|$ was discussed in great detail in the Appendix $\mathrm{C}$ of [13]. So, here we will refer to those equations (indicated as $(\mathcal{C}$.\#) in the following) and just point out the novelties or differences introduced by the presence of the twist $\mathrm{e}^{2 \pi \mathrm{i} \epsilon_{\mu} j_{0}^{\mu}}$ in the propagators.

The starting point is the tree-level vertex (2.8) with $N=2 g+N_{b}$ Hilbert spaces. We label the external Hilbert spaces with the index $I=1, \ldots, N_{b}$ and the legs to be sewn together in order to build the $g$ loops with the indices $2 \mu-1,2 \mu(\mu=1, \ldots, g)$. To be more precise we generate the higher genus surface, by identifying in the tree-level vertex $g$ pairs of Hilbert spaces: the Hilbert spaces $\mathcal{H}_{2 \mu-1}$ is identified with $\mathcal{H}_{2 \mu}$ by means of the projective transformation $P\left(x_{\mu}\right)$ and the twist operator $\mathrm{e}^{2 \pi \mathrm{i} \epsilon_{\mu} j_{0}^{\mu}}$. It is standard to indicate the local coordinates of the insertions by means of the functions $V_{i}(z)=\gamma_{i}(1-z)$ satisfying $V_{i}(0)=z_{i}$, see (2.8). $U_{i}(z)$ is related to the inverse of $V_{i}(z): U_{i}(z)=\Gamma V_{i}^{-1}(z)$, where $\Gamma$ is the inversion: $\Gamma(z)=1 / z$. As in [13], a tilde on $V_{2 \mu-1}$ or $U_{2 \mu-1}$ indicates the composition of the local coordinates with the projective transformation generated by the propagator $P: \tilde{V}=V P$. In the following formulae we will always write explicitly the effect of the twist induced by $\mathrm{e}^{2 \pi \mathrm{i} \epsilon j_{0}}$. The result of this manipulation is a generating vertex with $N_{b}$ insertions on the $g$-torus which generalizes ${ }^{8}$ Eq. $(\mathcal{C} .5)$

$$
\left\langle V_{N_{b} ; g}^{b c}\right|=\left[\prod_{I=1}^{N_{b}}\langle q=-Q|\right]\left\langle-Q_{g}\left|\Delta_{b} \exp \left\{\sum_{I=1}^{N_{b}} c_{\lambda}^{(I)} e_{I}\right\} \mathcal{M}\right|-Q_{g}\right\rangle,
$$

\footnotetext{
${ }^{8}$ The reader should keep in mind that in this paper we are interested only in the "minimal" correlation function (2.9) with $N_{b}$ insertions of $b$ field; thus in the following equation, we can ignore all the external oscillators $b_{n}^{(I)} \forall n$ and the $c_{n}^{(I)}$ for $n \geq \lambda+1$. In this respect our treatment is less general than the one in [13]. It is straightforward, although even more cumbersome, to discuss the twisted vertex $\left\langle V_{N_{b} ; g}^{b c}\right|$ in full generality.
} 
where

- $\left|-Q_{g}\right\rangle$ stays for $\prod_{\mu=1}^{g}|q=-Q\rangle_{(2 \mu)}$,

- $\Delta_{b}$ is the fermionic delta-function on the zero-modes of the internal lines already present at tree-level (2.8)

$$
\Delta_{b}=\prod_{r=1-\lambda}^{\lambda-1}\left\{\sum_{s=1-\lambda}^{\lambda-1} \sum_{\mu=1}^{g}\left[E_{r s}\left(\widetilde{V}_{2 \mu-1}\right) \mathrm{e}^{2 \pi \mathrm{i} \epsilon_{\mu}} b_{s}^{(2 \mu)}+\mathrm{e}^{-\mathrm{i} \pi \lambda} E_{r s}\left(V_{2 \mu}\right) b_{s}^{(2 \mu)}\right]\right\},
$$

- $E_{n s}(\gamma)$ are the matrices of the representation of the projective group with weight $\lambda[13]$,

- $e_{I}$ summarizes the coupling between the external lines and the zero-modes of the internal lines

$$
e_{I}=\mathrm{e}^{\mathrm{i} \pi(1-\lambda)} \sum_{\mu=1}^{g} \sum_{s=1-\lambda}^{\lambda-1}\left[E_{\lambda s}\left(U_{I} \widetilde{V}_{2 \mu-1}\right) \mathrm{e}^{2 \pi \mathrm{i} \epsilon_{\mu}} b_{s}^{(2 \mu)}+\mathrm{e}^{-\mathrm{i} \pi \lambda} E_{\lambda s}\left(U_{I} V_{2 \mu}\right) b_{-s}^{(2 \mu)}\right]
$$

- $\mathcal{M}$ is the result of the trace over the non-zero modes $(n \geq \lambda)$ of the internal lines, that we perform using coherent states. The computation is then reduced to a fermionic Gaussian integral and the effect of the twists $\mathrm{e}^{2 \pi \mathrm{i} \epsilon_{\mu} j_{0}^{\mu}}$ is to add some phases in the various coefficients of the Gaussian integral (C.9).

The result of the trace over the internal non-zero modes can be written in the usual form

$$
\mathcal{M}=\operatorname{det}(1-H) \exp \left[-\left(C_{1} C_{2}\right)(1-H)^{-1}\left(\begin{array}{c}
B_{1} \\
B_{2}
\end{array}\right)\right]
$$

with a slightly modified definition for the quadratic form $H$ and for the linear parts $C_{1}$, $B_{1}$ and $B_{2}\left(C_{2}\right.$ is unchanged $)$. It is simple to see that the new $H$ is like $(\mathcal{C} .9)$ with the first $g$ blocks of columns multiplied by $\mathrm{e}^{2 \pi \mathrm{i} \epsilon_{\nu}}$ and last $g$ blocks of rows multiplied by $\mathrm{e}^{-2 \pi \mathrm{i} \epsilon_{\mu}}$

$$
H_{n m}^{\mu \nu}=\left(\begin{array}{cc}
E_{n m}\left(U_{2 \mu} \widetilde{V}_{2 \nu-1}\right) \mathrm{e}^{2 \pi \mathrm{i} \epsilon_{\nu}} & \mathrm{e}^{-\pi \mathrm{i} \lambda} E_{n m}\left(U_{2 \mu} V_{2 \nu}\right) \\
\mathrm{e}^{-\pi \mathrm{i} \lambda} E_{n m}\left(\widetilde{U}_{2 \mu-1} \widetilde{V}_{2 \nu-1}\right) \mathrm{e}^{2 \pi \mathrm{i}\left(\epsilon_{\nu}-\epsilon_{\mu}\right)} & E_{n m}\left(\widetilde{U}_{2 \mu-1} V_{2 \nu}\right) \mathrm{e}^{-2 \pi \mathrm{i}\left(\epsilon_{\mu}+\lambda\right)}
\end{array}\right)
$$

whith $n, m \geq \lambda, \mu, \nu=1, \ldots, g$; moreover in the off-diagonal entries we used the conven- 
tion $E_{n m}\left(U_{2 \mu} V_{2 \mu}\right)=E_{n m}\left(\widetilde{U}_{2 \mu-1} \widetilde{V}_{2 \mu-1}\right)=0, \forall n, m$. The new linear terms are

$$
\begin{aligned}
\left(C_{1}\right)_{m}^{\mu} & =\mathrm{e}^{\pi \mathrm{i}(1-\lambda)} \sum_{I=1}^{N} c_{\lambda}^{(I)} E_{\lambda m}\left(U_{I} \widetilde{V}_{2 \mu-1}\right) \mathrm{e}^{2 \pi \mathrm{i} \epsilon_{\mu}} \\
\left(C_{2}\right)_{m}^{\mu} & =-\mathrm{e}^{2 \pi \mathrm{i} \lambda} \sum_{I=1}^{N} c_{\lambda}^{(I)} E_{\lambda m}\left(U_{I} V_{2 \mu}\right) \\
\left(B_{1}\right)_{n}^{\mu} & =-\sum_{\nu=1}^{g} \sum_{r=1-\lambda}^{\lambda-1}\left[E_{n r}\left(U_{2 \mu} \widetilde{V}_{2 \nu-1}\right) b_{r}^{(2 \nu)} \mathrm{e}^{2 \pi \mathrm{i} \epsilon_{\nu}}+\mathrm{e}^{-\pi \mathrm{i} \lambda} E_{n r}\left(U_{2 \mu} \widetilde{V}_{2 \nu}\right) b_{-r}^{(2 \nu)}\right] \\
\left(B_{2}\right)_{n}^{\mu} & =\mathrm{e}^{\pi \mathrm{i}(1-\lambda)} \mathrm{e}^{-2 \pi \mathrm{i} \epsilon_{\mu}} \sum_{\nu=1}^{g} \sum_{r=1-\lambda}^{\lambda-1}\left[E_{n r}\left(\widetilde{U}_{2 \mu-1} \widetilde{V}_{2 \nu-1}\right) \mathrm{e}^{2 \pi \mathrm{i} \epsilon_{\nu}} b_{r}^{(2 \nu)}+E_{n r}\left(\widetilde{U}_{2 \mu-1} V_{2 \nu}\right) b_{-r}^{(2 \nu)}\right] .
\end{aligned}
$$

Again we have neglected the terms containing $c_{n}^{(I)}, n \geq \lambda+1$ and all $b^{(I)}$ which are present in the complete expressions of $\left(B_{1}\right)$ and $\left(B_{2}\right)$.

It is important to notice that the presence of the twists along the $b$-cycles does not modify the Schottky group structure present in [13]: the building blocks of the computation are still written in terms of the $E^{\lambda}$ representation of the projective group, and the twists appear only as a multiplicative phase of the usual $E^{\lambda}$ matrices. In particular this means that in the calculation of $\mathcal{M}$ one still reconstructs the $g$ Schottky generators through the usual combination of local coordinates and the untwisted propagator

$$
S_{\mu}=\widetilde{V}_{2 \mu-1} U_{2 \mu}=V_{2 \mu-1} P \Gamma V_{2 \mu}^{-1} .
$$

Because of this reason, it is not difficult to see, by following appendices E and D of [12], that $\operatorname{det}(1-H)$ is exactly given by Eq. (2.11).

In order to compute the zero-mode contribution (i.e. the expectation value in (C.1)), it is necessary to expand the factor of $(1-H)^{-1}$ in the exponent of (C.4) in powers of $H$. Following the same steps of [13] one gets

$$
\exp \left\{\sum_{I=1}^{N_{b}} c_{\lambda}^{(I)} e_{I}\right\} \mathcal{M}=\operatorname{det}(1-H) \exp \left[\sum_{I=1}^{N_{b}} c_{\lambda}^{(I)} f_{I}\right]
$$

where

$$
f_{I}=\mathrm{e}^{-2 i \pi \lambda} \sum_{\alpha} \sum_{\mu=1}^{g} \sum_{m=\lambda}^{\infty} \sum_{r, s=1-\lambda}^{\lambda-1} E_{\lambda m}\left(U_{I} T_{\alpha}\right) E_{m r}\left(S_{\mu}\right) E_{r s}\left(U_{\mu}\right) \mathrm{e}^{2 \pi \mathrm{i}\left(\epsilon \cdot N_{\alpha}+\epsilon_{\mu}\right)} b_{-s}^{(2 \mu)},
$$

where the sum $\sum_{\alpha}$ is extended over all the elements $T_{\alpha}$ of the Schottky group $\mathcal{S}_{g}$. The operator $\mathcal{F}_{N_{b}, g}$ in Eq. (2.10) is then given by

$$
\mathcal{F}_{N_{b}, g}=\left\langle-Q_{g}\left|\Delta_{b} \exp \left[\sum_{I=1}^{N_{b}} c_{\lambda}^{(I)} f_{I}\right]\right|-Q_{g}\right\rangle
$$


The explicit evaluation of this equation is particularly simple in the case $\lambda=1$, where it is directly related to the twisted abelian differential (2.18). First, the sums and the products on $r, s=1-\lambda, \ldots, \lambda-1$ have a single term $r, s=0$; moreover from the explicit form of the representation $E$, see $\left(\mathcal{A}\right.$.1) of [13], it is easy to see that $E_{00}(\gamma)=1$ for all the projective transformations $\gamma$. Thus Eq. (C.2) simply becomes $\Delta_{b}=\sum_{\mu=1}^{g}\left(\mathrm{e}^{2 \pi \mathrm{i} \epsilon_{\mu}}-1\right) b_{0}^{(2 \mu)}$, while the sum $\sum_{m} E_{1 m}\left(U_{I} T_{\alpha}\right) E_{m 0}\left(S_{\mu}\right)$ present in $f_{I}$ (C.9) can be treated as done in Appendix D of $[13]^{9}$

$$
\begin{aligned}
\sum_{m=1}^{\infty} E_{1 m}\left(U_{I} T_{\alpha}\right) E_{m 0}\left(S_{\mu}\right) & =-\left(T_{\alpha}^{\prime}\right)^{-1}\left(z_{I}\right)\left[\frac{1}{T_{\alpha}^{-1}\left(z_{I}\right)-S_{\mu}(0)}-\frac{1}{T_{\alpha}^{-1}\left(z_{I}\right)}\right] \\
& =-\left[\frac{1}{z_{I}-T_{\alpha} S_{\mu}(0)}-\frac{1}{z_{I}-T_{\alpha}(0)}\right]
\end{aligned}
$$

The first identity follows from Eq. $(\mathcal{A}$.11) of [13] and from the simplest possible choice of the local coordinates $V_{I}(z)=z_{I}-z$, while the second line is obtained by using (B.8). At this point one can easily compute the scalar product in the $g$ Hilbert spaces of the loops (C.10) and in the $g-1$ Hilbert spaces of external fields (2.13), simply by recalling that $\left\langle q=1\left|b_{0}\right| q=1\right\rangle=1$. This means that one has to select a factor of $b_{0}$ for each one of the $\mu=1, \ldots, g$ Hilbert spaces $\mathcal{H}_{\mu}$ and one $c_{1}^{(I)}$ for each of external Hilbert space $\mathcal{H}^{(I)}$ : this yields the determinant (2.14). Then a slightly non-trivial step is to show that the constituents of this determinant can be written in the form of Eq. (2.19). The basic idea is to single out the sum over the elements of the form $T_{\alpha} S_{\mu}^{\ell}$. For sake of notational simplicity, we use the following abbreviations $x=\exp \left(2 \pi \mathrm{i} \epsilon_{\mu}\right)$ and $c_{\ell}=1 /\left(z-T_{\alpha} S_{\mu}^{\ell}(0)\right)$, so Eq. (2.15) can be rewritten as

$$
\zeta_{\mu}\left(z_{I}\right)=\sum_{\alpha}^{(\mu)} x \sum_{\ell=-\infty}^{\ell=\infty} x^{\ell}\left(c_{\ell+1}-c_{\ell}\right)
$$

Let us focus on the series over $\ell$ in the parenthesis and relabel the summed index in order to combine the two terms of (C.12)

$\lim _{N_{1}, N_{2} \rightarrow \infty} \sum_{\ell=-N_{2}}^{N_{1}} x^{\ell}\left(c_{\ell+1}-c_{\ell}\right)=\lim _{N_{1}, N_{2} \rightarrow \infty}\left\{(1-x) \sum_{\ell=-N_{2}+1}^{N_{1}} c_{\ell} x^{\ell-1}+x^{N_{1}} c_{N_{1}+1}-x^{-N_{2}} c_{-N_{2}}\right\}$.

The convergence of the series is made manifest by breaking the sum into two pieces $(\ell>0$ and $\ell \leq 0)$ and by adding ad subtracting the limiting values

$$
A=\lim _{\ell \rightarrow \infty} c_{\ell}=\frac{1}{z-T_{\alpha}\left(\eta_{\mu}\right)} \quad, \quad R=\lim _{\ell \rightarrow-\infty} c_{\ell}=\frac{1}{z-T_{\alpha}\left(\xi_{\mu}\right)} .
$$

\footnotetext{
${ }^{9}$ We refer in particular to Eq. $(\mathcal{D} .2)$. Notice that here we present a generalization of that identity, since (C.11) is valid for all projective transformation $T_{\alpha}$, without the need of the sum over all the element of the Schottky group $\sum_{\alpha}$.
} 
So the parenthesis in (C.12) can be rewritten as follows

$$
\begin{aligned}
\{\ldots\}=\lim _{N_{1}, N_{2} \rightarrow \infty}\left\{(1-x) \sum_{\ell=1}^{N_{1}} x^{\ell-1}\left(c_{\ell}-A\right)+\left(1-x^{N_{1}}\right) A+x^{N_{1}} c_{N_{1}+1}+\right. \\
\left.(1-x) \sum_{\ell=0}^{N_{2}-1} x^{-\ell-1}\left(c_{-\ell}-R\right)-\left(1-x^{-N_{2}}\right) R-x^{-N_{2}} c_{-N_{2}}\right\} .
\end{aligned}
$$

Thanks to (C.14) the terms proportional to $x^{N_{i}}$ cancel in the limit and one obtains

$$
\{\ldots\}=A-R-\left(1-x^{-1}\right)\left[\sum_{\ell=1}^{\infty} x^{\ell}\left(c_{\ell}-A\right)+\sum_{\ell=0}^{\infty} x^{-\ell}\left(c_{-\ell}-R\right)\right] .
$$

When this result, together with (C.14), is used in (C.12), the first two terms give exactly the first line of (2.19), while the second line of (2.19) is reproduced by the square parenthesis.

In the case $\lambda=3 / 2,2, \ldots$ the computations are more cumbersome because we have $(2 \lambda-1)$ zero-modes and then the number of insertions necessary to have a non-trivial result is $N_{b}=(2 \lambda-1)(g-1)$. Similarly to what has just been done for $\lambda=1$, one can start from (C.10) and derive $\mathcal{F}^{(\lambda)}$ which is the generalization of (2.13) for a generic spin $\lambda$. It is convenient to replace the index $I=1, \ldots, N_{b}$ with a double index $(\mu, r)$ with $\mu=1, \ldots, g-1$ and $r=1-\lambda, \ldots, \lambda-1$. With this notation one gets

$$
\mathcal{F}^{(\lambda)}=\operatorname{det}\left(\begin{array}{ccc}
\boldsymbol{\zeta}_{1}\left(\boldsymbol{z}_{1}\right) & \ldots & \boldsymbol{\zeta}_{g}\left(\boldsymbol{z}_{1}\right) \\
\vdots & & \vdots \\
\boldsymbol{\zeta}_{1}\left(\boldsymbol{z}_{g-1}\right) & \ldots & \boldsymbol{\zeta}_{g}\left(\boldsymbol{z}_{g-1}\right) \\
\mathcal{E}\left(S_{1}\right) & \ldots & \mathcal{E}\left(S_{g}\right)
\end{array}\right)
$$

where $\mathcal{E}\left(S_{\mu}\right)_{r s}$ is defined in (2.22) and where each entry of (C.17) is a $(2 \lambda-1) \times(2 \lambda-1)$ matrix $\left[\boldsymbol{\zeta}_{\boldsymbol{\mu}}\left(\boldsymbol{z}_{\nu}\right)\right]_{r s}=\zeta_{\mu, s}\left(z_{\nu, r}\right)$

$$
\zeta_{\mu, r}(z)=\left.\sum_{\alpha} \mathrm{e}^{-2 \pi \mathrm{i}\left(\epsilon \cdot N_{\alpha}-\epsilon_{\mu}\right)} \frac{\partial_{y}^{(r+\lambda-1)}}{(r+\lambda-1) !} \frac{\left(T_{\alpha}^{\prime}(z)\right)^{\lambda}\left(S_{\mu}^{\prime}(y)\right)^{1-\lambda}}{T_{\alpha}(z)-S_{\mu}(y)}\left(\frac{S_{\mu}(y)}{T_{\alpha}(z)}\right)^{2 \lambda-1}\right|_{y=0}
$$

The convergence of the Poincaré series which defines $\zeta$ is assured by the factor $\left(T_{\alpha}^{\prime}(z)\right)^{\lambda}$. In fact it is easy to show that $T_{\alpha}^{\prime}(z)=k_{\alpha}\left(T_{\alpha}(z)-\xi_{\alpha}\right)^{2} /\left(z-\xi_{\alpha}\right)^{2}$. Moreover, the multiplier of a composite transformation $T_{\alpha}$ depends on the multipliers (and the fixed points) of the generators present in $T_{\alpha}$; in particular $k_{\alpha}$ contains a factor $k_{\mu}^{l}$, if the generator $S_{\mu}$ or its inverse appear $l$ times in the expression of $T_{\alpha}$. Thus when the order $\alpha$ increases, the element in the sum (C.18) contains high powers of some multiplier, thanks to the factor $\left(T_{\alpha}^{\prime}(z)\right)^{\lambda}$. Since $|k|<1$ the only condition for the convergence of the series is $\lambda>0$. 
It is quite easy to check that for $\lambda=1$ the determinant (C.17) reduces to the result in Eq. (2.14). In fact

$$
\begin{aligned}
\zeta_{\mu}(z) d z & =\sum_{\alpha} \mathrm{e}^{-2 \pi \mathrm{i}\left(\epsilon \cdot N_{\alpha}-\epsilon_{\mu}\right)} \frac{S_{\mu}(0) d T_{\alpha}(z)}{\left(T_{\alpha}(z)-S_{\mu}(0)\right) T_{\alpha}(z)} \\
& =\sum_{\alpha} \mathrm{e}^{-2 \pi \mathrm{i}\left(\epsilon \cdot N_{\alpha}-\epsilon_{\mu}\right)} d \log \frac{T_{\alpha}(z)-S_{\mu}(0)}{T_{\alpha}(z)} \\
& =\sum_{\alpha} \mathrm{e}^{2 \pi \mathrm{i}\left(\epsilon \cdot N_{\alpha}+\epsilon_{\mu}\right)}\left[\frac{1}{z-T_{\alpha} S_{\mu}(0)}-\frac{1}{z-T_{\alpha}(0)}\right] d z .
\end{aligned}
$$

where in the last line we used Eq. (B.8) and then relabeled $T_{\alpha}^{-1}$ as $T_{\alpha}$ in the sum. In the general case $\lambda=3 / 2,2, \ldots$, the analysis is qualitatively similar to the $\lambda=1$ case described in Section 3. On one hand the $\zeta_{\mu, r}$ defined in (C.18) have the expected periodicity properties, since they are single valued when $z$ is moved around an $a$-cycle, while around a $b_{\nu}$-cycle they transform as

$$
\zeta_{\mu, r}\left(S_{\nu}(z)\right)\left(d S_{\nu}(z)\right)^{\lambda}=\mathrm{e}^{2 \pi \mathrm{i} \epsilon_{\nu}} \zeta_{\mu, r}(z)(d z)^{\lambda} .
$$

However, the $\zeta_{\mu, r}$ in (C.18) are too many to be identified with the twisted $\lambda$-differentials, since they are $(2 \lambda-1) g$, instead of $(2 \lambda-1)(g-1)$, as expected. In fact this identification is not possible, since the $\zeta_{\mu, r}$ are not holomorphic, because of the pole singularity in $z=0$. The singular part comes only from two elements of the Poincaré series (C.18): $T_{\alpha}=I$ and $T_{\alpha}=S_{\mu}$. So it is easy to extract this singularity for $z \rightarrow 0$

$$
\zeta_{\mu, r}(z) \sim-\sum_{s=1-\lambda}^{\lambda-1} \frac{1}{z^{s+\lambda}} \mathcal{E}\left(S_{\mu}\right)_{s r}
$$

Therefore one can build $(2 \lambda-1)(g-1)$ twisted holomorphic differentials by generalizing Eq. (2.18)

$$
\Omega_{\mu, r}(z)=\left[\zeta_{\mu, r}(z)-\sum_{s, t=1-\lambda}^{\lambda-1} \zeta_{g, s}(z)\left(\mathcal{E}\left(S_{g}\right)\right)_{s t}^{-1} \mathcal{E}\left(S_{\mu}\right)_{t r}\right] \quad, \quad \mu=1, \ldots, g-1 .
$$

In order to write the $\Omega$ 's we have of course supposed that $\mathcal{E}\left(S_{g}\right)$ is invertible which is surely true for any $\lambda$ if $\epsilon_{g} \neq 0$. In fact one can easily diagonalize the $(2 l-1) \times(2 \lambda-1)$ matrix $\mathcal{E}\left(S_{g}\right)$ and check that its eigenvalues are

$$
\left(\mathrm{e}^{2 \pi \mathrm{i} \epsilon_{g}} k_{g}^{r}-1\right), \text { with } r=1-\lambda, \ldots, \lambda-1,
$$

and none of them is vanishing. As for $\lambda=1$ (2.17), one can write (C.17) in terms of these $\lambda$-differentials

$$
\mathcal{F}^{(\lambda)}=\operatorname{det}\left(\begin{array}{cccc}
\boldsymbol{\Omega}_{1}\left(\boldsymbol{z}_{1}\right) & \ldots & \boldsymbol{\Omega}_{g-1}\left(\boldsymbol{z}_{1}\right) & \boldsymbol{\zeta}_{g}\left(\boldsymbol{z}_{1}\right) \\
\vdots & & \vdots & \vdots \\
\boldsymbol{\Omega}_{1}\left(\boldsymbol{z}_{g-1}\right) & \ldots & \boldsymbol{\Omega}_{g-1}\left(\boldsymbol{z}_{g-1}\right) & \boldsymbol{\zeta}_{g}\left(\boldsymbol{z}_{g-1}\right) \\
0 & \ldots & 0 & \mathcal{E}\left(S_{g}\right)
\end{array}\right)
$$


and the final result for the "minimal" correlator we are interested in is given by the product of (2.11) and (C.24): $Z_{\epsilon}^{\lambda}=\operatorname{det}(1-H) \mathcal{F}^{(\lambda)}$.

For half-integer $\lambda(\lambda=3 / 2,5 / 2, \ldots)$, the same computation can be done also for the case where $\epsilon_{\mu}=0 \forall \mu$. In fact, all the matrices $\mathcal{E}\left(S_{\mu}\right)$ are still invertible also in this case. This can be easily seen from (C.23) which tells that for half-integer $r$ the eigenvalues of any $\mathcal{E}(S)$ are non-vanishing even if $\epsilon=0$. In order to compare with the result of [13], one can show that the differential $\Lambda_{\mu}(z)$ introduced there, see Eq. $(\mathcal{D} .12)$, can be derived also starting from the $\zeta_{\mu}$ defined in (C.18) setting $\epsilon_{\mu}=0 \forall \mu$

$$
\Lambda_{\mu}(z)=-\zeta_{\mu} \mathcal{E}\left(S_{\mu}\right)^{-1}+\zeta_{g} \mathcal{E}\left(S_{g}\right)^{-1}
$$

This means that for half-integer $\lambda$ the twists along the $b$-cycles do not introduce any qualitative change in the geometric interpretation of the final result and their effect is just to bring some phase factor in the explicit definition of the differentials. This is to be contrasted with the $\lambda=1$ case where the counting of the zero-modes of the $\bar{\partial}$ operator changes in presence of non-trivial twists.

The case $\lambda=1 / 2$ is special $[14,15,16]$ since all the zero-modes are absent. Of course one can still write a Poincaré series similar to (C.18)

$$
\sum_{\alpha} \mathrm{e}^{-2 \pi \mathrm{i}\left(\epsilon \cdot N_{\alpha}-\epsilon_{\mu}\right)} \frac{\left(T_{\alpha}^{\prime}(z)\right)^{1 / 2}\left(S_{\mu}^{\prime}(0)\right)^{1 / 2}}{T_{\alpha}(z)-S_{\mu}(0)},
$$

which is an automorphic form of weight 1/2 closely related to the Szëgo kernel. However it is not possible to use the trick in (C.25) and eliminate the pole in $z=0$ in order obtain some non-trivial holomorphic differentials. In fact, the substitution $T_{\alpha}=S_{\mu} T_{\beta}$ shows that (C.26) does not depend on $\mu$ and thus subtracting two values of $\mu$ in (C.26), as done in (C.25), one obtains identically zero.

For integer $\lambda \geq 2$ and vanishing twists $\epsilon=0$, we have to distinguish two cases. On the torus $g=1$ both $b$ and $c$ have a zero-mode, see Eq. (2.5). On higher genus surfaces $g \geq 2$, only $b$ has zero-modes. Thus, for higher genus surfaces, the $\epsilon=0$ case is conceptually on the same footing as the twisted case and one can build $(2 \lambda-1)(g-1)$ differentials starting from (C.18). First it is easy to see why the zero-modes of $c$ are absent in this case. Usually they are constructed starting from the $\zeta_{\mu, r}$ in (C.18) by sending $\lambda \rightarrow 1-\lambda$. Formally this satisfies the periodicity condition, but the series does not converge since the factor of $T_{\alpha}^{\prime}(z)$ has now a negative power. Since there are no zero-modes for $c$, the determinant (C.17) does not vanish even if $\operatorname{det}\left[\mathcal{E}\left(S_{\mu}\right)\right]=0 \forall \mu$ when $\epsilon=0$. Hence the Riemann-Roch theorem for $g \geq 2$ is realized in the same way, independently of possible twists for $\lambda=3 / 2,2, \ldots$.

\section{References}

[1] V. Alessandrini and D. Amati, Nuovo Cim. A 4 (1971) 793. 
[2] A. M. Polyakov, Phys. Lett. B 103 (1981) 207.

D. Friedan, E. J. Martinec and S. H. Shenker, Nucl. Phys. B 271 (1986) 93.

[3] V. G. Knizhnik, Phys. Lett. B 180 (1986) 247.

[4] L. Alvarez-Gaume, G. W. Moore and C. Vafa, Commun. Math. Phys. 106 (1986) 1.

[5] E. Verlinde and H. Verlinde, Nucl. Phys. B 288 (1987) 357.

[6] L. Alvarez-Gaume, J. B. Bost, G. W. Moore, P. Nelson and C. Vafa, Commun. Math. Phys. 112 (1987) 503.

[7] T. Eguchi and H. Ooguri, Phys. Lett. B 187 (1987) 127.

[8] O. Lechtenfeld, Phys. Lett. B 232 (1989) 193.

[9] J. D. Fay, "Theta Functions on Riemann Surfaces" Springer Notes in Math. (1973).

[10] D. Mumford, "Tata lectures on Theta" Birkhauser (1983).

[11] J. D. Fay, "Kernel Functions Analytic Torsion and Moduli Spaces" Mem. AMS 96 464 (1992).

[12] P. Di Vecchia, F. Pezzella, M. Frau, K. Hornfeck, A. Lerda and A. Sciuto, Nucl. Phys. B 322 (1989) 317.

[13] P. Di Vecchia, F. Pezzella, M. Frau, K. Hornfeck, A. Lerda and S. Sciuto, Nucl. Phys. B 333 (1990) 635.

[14] F. Pezzella, Phys. Lett. B 220 (1989) 544.

[15] A. Losev, JETP Lett. 49 (1989) 424 [Pisma Zh. Eksp. Teor. Fiz. 49 (1989) 372].

[16] P. Di Vecchia, NORDITA-89/30-P In *Brink, L. (ed.) et al.: Physics and mathematics of strings* 212-230.

[17] C. S. Chu, R. Russo and S. Sciuto, Fortsch. Phys. 50 (2002) 871 [arXiv:hepth/0201118].

[18] M. B. Green, J. H. Schwarz and E. Witten, "Superstring Theory" Cambridge University Press (1987).

[19] S. Sciuto, Lett. Nuovo Cim. 2 (1969) 411.

A. Della Selva and S. Saito, Lett. Nuovo Cim. 4 (1970), 689.

[20] A. Neveu and P. C. West, Nucl. Phys. B 278 (1986) 601.

P. Di Vecchia, R. Nakayama, J. L. Petersen and S. Sciuto, Nucl. Phys. B 282 (1987) 103. 
[21] J. Polchinski, "String Theory. Vol. 1" Cambridge University Press (1998).

[22] C.-S. Chu, R. Russo and S. Sciuto, work in progress

[23] M. Frau, I. Pesando, S. Sciuto, A. Lerda and R. Russo, Phys. Lett. B 400 (1997) 52 [arXiv:hep-th/9702037].

[24] C. Bachas and M. Porrati, Phys. Lett. B 296 (1992) 77 [arXiv:hep-th/9209032].

[25] M. Billo, P. Di Vecchia and D. Cangemi, Phys. Lett. B 400 (1997) 63 [arXiv:hepth/9701190].

[26] A. Frizzo, L. Magnea and R. Russo, Nucl. Phys. B 604 (2001) 92 [arXiv:hep$\mathrm{ph} / 0012129]$.

[27] M. Billò, F. Lonegro and I. Pesando, personal communication.

[28] P. Di Vecchia, A. Liccardo, R. Marotta and F. Pezzella, [arXiv:hep-th/0305061]. 